\title{
Is balance impaired by recurrent sprained ankle?
}

\author{
Eli Isakov, J Mizrahi
}

\begin{abstract}
Objective-To evaluate uninjured and recurrent sprained ankles during single leg standing, both with and without visual input, and the contribution of related proprioceptive feedback in this event.

Methods-A force measuring system was used for monitoring reaction forces in the anteroposterior and mediolateral directions during single leg standing. Differences between selected variables obtained in the uninjured and sprained ankles were analysed using two way analysis of variance.

Results-Foot-ground reaction forces in both anteroposterior and mediolateral directions were the same in normal and sprained ankles of each subject while standing with either open or closed eyes. However, standing with closed eyes, irrespective of the ankle status, always produced significantly higher reaction forces than those obtained with open eyes $(P<0.05)$.

Conclusions-The amount of postural sway during single leg standing is similar in the chronically sprained and the uninjured ankle joint.

(Br F Sports Med 1997;31:65-67)
\end{abstract}

Biomechanics

Laboratory,

Loewenstein

Rehabilitation

Hospital, Tel-Aviv

University Medical

School, Ra'anana,

Israel

E Isakov

\section{Department of}

Biomedical

Engineering and Julius

Silver Institute of

Biomedical

Engineering,

Technion-Israel

Institute of Technology,

Haifa, Israel

J Mizrahi

Correspondence to:

Dr Eli Isakov, Head,

Orthopaedic Rehabilitation

Department, Loewenstein

Rehabilitation Hospital,

Ra'anana 43100, Israel.

Accepted for publication 12 September 1996 Inversion ankle sprain is one of the most common injuries among athletes. ${ }^{1}$ This condition is especially frustrating among athletes who are forced into long periods of partial or complete inactivity. A large variety of corrective surgical ${ }^{2}$ and prophylactic conservative treatments have been suggested for this pathology. ${ }^{3}$

The stabilising elements of the normal ankle are the capsular and ligamentous structures, the musculotendinous units, and the anatomical alignment of the osseous components of the joint. Stability of the ankle joint during functional activities such as standing, walking, and running, exists in the presence of intact neural input from proprioceptors in the joint capsules, ligaments, muscles, tendons, and skin. Proprioceptive information is generated by ligamentous mechanoreceptors, Golgi tendon organs, and muscle spindles, which transduce mechanical distortion of the surrounding connective tissue. ${ }^{45}$ Traumatic ankle inversion often results in capsular and ligamentous damage with concomitant damage to the joint mechanoreceptors on the lateral side of the talocrural and subtalar joints. ${ }^{67}$ Unfortunately, an inversion sprain leads to a chronically symptomatic or unstable ankle in approximately $40 \%$ of cases. ${ }^{89}$

Two different theories have been suggested to explain recurrent ankle sprains. ${ }^{1011}$ The first is the functional instability theory, which assumes that the afferent nerve fibres in the capsule, ligaments, and muscles subserve reflexes that help to stabilise the ankle. The second is the mechanical instability theory, which assumes that instability is due to a loss of integrity in the supporting ligaments of the ankle complex. The result is a pathological anterior drawer sign. ${ }^{3}$ However, no consistent relation has been found between functional and mechanical instability. Assuming that ankle stability depends on intact peripheral afferent proprioceptive input, ${ }^{812}$ the reaction time of the peroneal muscles to a sudden inversion tilt in recurrently sprained and in normal ankles was measured. The results indicated that the reaction time of these muscles in the injured ankle was significantly slower. These findings were disputed by others ${ }^{13}$ who conducted a similar measurement procedure and found an almost equal reaction time in both peroneal muscles.

The ability to maintain balance during standing on a single leg or both legs depends on the integrity of the visual, vestibular, and nervous systems. In the presence of an intact vestibular system, standing with eyes closed depends mostly on the normal function of the various proprioceptive receptors. ${ }^{414}$ An inversion ankle sprain that results in injury to the proprioreceptors of capsule and ligaments may impair standing balance on the affected limb, especially when eyes are closed. ${ }^{81114}$

Several research methods are used to assess ankle proprioception during upright standing. The modified Romberg test, ${ }^{15}$ measurements of displacement of the centre of foot pressure, ${ }^{16}$ and the adjustment reactions to balance perturbation induced by tilting of the support surface. ${ }^{12} 17$ The purpose of this study was to investigate whether stability control of the ankle measured by ground reaction forces during single leg stance is impaired in gymnasts with a unilateral chronically sprained ankle. 


\section{Methods}

Eight competitive female gymnasts of mean age of 16.2 years (range 14 to 18 years) were included in this study.

All subjects, with right lower limb dominance, reported a past history of at least three inversion injuries to one ankle only, which required protected weightbearing and immobilisation. A manual evaluation for anteroposterior stability of each ankle was performed. In all eight affected ankles, the anterior drawer sign was positive, as shown by the greater forward excursion of the injured compared to the uninjured foot. Four suffered right ankle sprains; four left ankle recurrent sprains. The sprains were never accompanied by a fracture of the ankle osseous elements. During the four months before testing, all subjects were fully weightbearing, pain-free, and the functional use of the ankle was unimpaired.

\section{EQUIPMENT}

Postural sway was evaluated by means of a force measuring system, which consisted of a force plate (Kistler Z-4305, Winterthur, Switzerland). The foot-ground reaction forces in the anteroposterior and mediolateral directions were monitored on-line for each foot during the test. These are the tangential components of the force signal responsible to maintain equilibrium during standing and exerted by the body at the foot level. The time variation in these forces reflects the swaying motion of the body in the horizontal plane. The force signals from the Kistler amplifier (type 9803), with gains set at 5 Newton volt ${ }^{-1}$ for the mediolateral and anteroposterior directions, were routed to a computer through a multichannel analogue to digital converter at a sampling rate of $50 \mathrm{~Hz}$.

The force traces obtained included transient, slow (approximately $0.1 \mathrm{~Hz}$ ) upon which more rapid ( $1 \mathrm{~Hz}$ and higher) oscillations were superimposed. A numerical procedure was set to rectify these rapid oscillations and to compute their average amplitudes. ${ }^{14}$ The baseline of the rapid oscillations was established by computing the point by point moving average of the signal. The computation was based on 100 samples, 50 on each side of the time point. To allow comparison between the different tests and subjects, the averages obtained were normalised to the body weight of each of the subjects and expressed as a percentage of body weight $(\% \mathrm{~W})$.

\section{PROCEDURE}

Before testing all subjects received a thorough clinical orthopaedic examination. No gross swelling, bony asymmetry, points of tenderness, or deficits in range of motion were found.

Each test lasted 35 seconds, of which only the middle 25 seconds were analysed to avoid the "edge effect" of the test. Recordings were made in two positions. The first was standing on the right leg with the left leg raised and flexed at the knee and the second repeated the test on the opposite side (figure). The subjects were tested in two conditions: with eyes open, gazing at a fixed target three metres ahead, at eye level. The second test was done with eyes closed. During

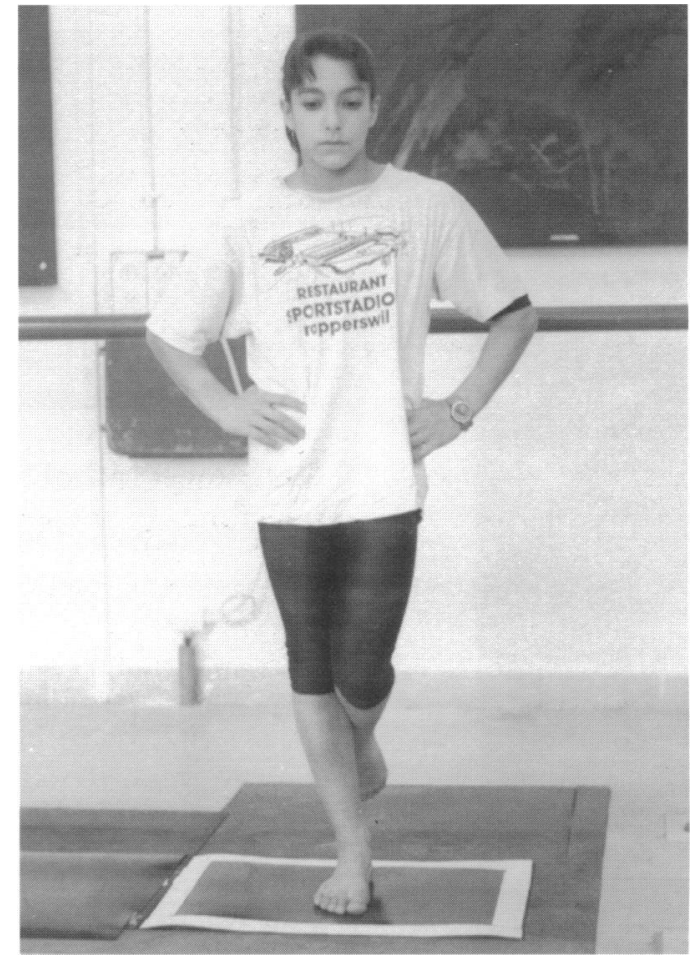

Subject standing on a force plate.

the test, the subject was not allowed to move the foot from the initial position. The arms (hands resting on waist) and lifted leg were not restricted, so as to allow compensatory movements whenever necessary. Since in most tests such movements were minor it was concluded that their effect on the force records were negligible. Each test was performed three times and the average was taken.

Two way analysis of variance (ANOVA) was used to confirm the significant changes for selected variables between uninjured and sprained ankles and between standing with open and closed eyes. Results were considered to be statistically significant at $\mathrm{P}<0.05$.

\section{Results}

The results are detailed in the table. Ground reaction forces measured in the uninjured and sprained ankle limbs were compared while standing with open and closed eyes. In the mediolateral direction, values ( $\% \mathrm{~W}$ ) in the uninjured and sprained ankle with eyes open were 0.328 and 0.301 , respectively $(F=0.36$; $P>0.05)$, and with closed eyes 0.111 and 0.951 , respectively $(F=1.18 ; P>0.05)$. In the anteroposterior direction, values in the uninjured and sprained ankle with eyes open were 0.240 and 0.235 , respectively $(F=0.02 ; P>$ $0.05)$, and when eyes closed 0.925 and 0.699 , respectively $(\mathrm{F}=1.07 ; \mathrm{P}>0.05)$.

Comparison between the open and closed eyes positions yielded the following significant differences $(P<0.05)$ : for the uninjured limb, the $F$ value was 12.26 in the mediolateral direction and 8.94 in the anteroposterior directions; for the affected limb, the $F$ value was 29.73 in the mediolateral directions and 15.07 in the anteroposterior directions. 
Summary of means (SD) and two way analysis of variance of foot-ground forces (\% body weight) obtained during single leg standing

\begin{tabular}{|c|c|c|c|c|c|c|}
\hline \multirow[b]{2}{*}{ Eye position } & \multicolumn{3}{|c|}{ Mediolateral force } & \multicolumn{3}{|c|}{ Anterposterior force } \\
\hline & $\begin{array}{l}\text { Uninjured } \\
\text { ankle }\end{array}$ & Sprained ankle & $F$ & Uninjured ankle & Sprained ankle & $F$ \\
\hline Open & $0.328(0.999)$ & $0.301(0.115)$ & 0.36 & $0.240(0.090)$ & $0.235(0.066)$ & 0.02 \\
\hline Closed & $1.111(0.625)$ & $0.951(0.438)$ & 1.18 & $0.925(0.675)$ & $0.699(0.379)$ & 1.07 \\
\hline $\mathbf{F}$ & $\star 12.26$ & $\star 29.73$ & & $\star 8.94$ & $\star 15.07$ & \\
\hline
\end{tabular}

*Significant at $\mathrm{P}<0.05$.

\section{Discussion}

The results obtained in this study show that during single leg standing on an uninjured and a recurrently sprained ankle the tangential ground reaction forces are not significantly different between the two legs in either the mediolateral or the anteroposterior directions. Comparison between the results obtained while standing with open and closed eyes indicates significantly higher tangential ground reaction forces with closed eyes. Limb dominance had no effect on these results since no significant differences were found between left and right single leg standing either with open or with closed eyes. ${ }^{18}$ These results suggest that the presence of intact joint proprioception is required for single leg standing with open or closed eyes, and that the normal activity of the muscles involved in balance maintenance is not impaired in the affected ankle joint.

In 1986 Isakov et al ${ }^{13}$ measured the peroneal reaction time during sudden inversion in uninjured and recurrently sprained ankles. The latency times of the peroneal muscles were measured in a group of healthy subjects and in a second group of subjects suffering from recurrent ankle sprains. Peroneal latency times in healthy subjects were 69.3 (SD 6.4) and 67 (5.4) $\mathrm{ms}$ in the left and right ankle respectively, and $70.2(7.4)$ and $68.3(6.5) \mathrm{ms}$ in the sprained and sound ankle respectively in the second group. These results also showed that differences between affected and uninjured ankles were insignificant.

The results in both the present work and in the 1986 study $^{13}$ support the findings of a recent study which investigated the effect on proprioception during experimental suppression of the afferent information from the ligaments and capsule by anaesthetising the ankle and foot in normal subjects. Results obtained indicate that the magnitude of postural sway during single leg stance was unchanged by anaesthesia of the ankle and foot and that the peroneal reaction time to sudden ankle inversion was not altered. ${ }^{19}$

The presence of mechanoreceptors in the joint capsule and ligaments is well documented and it seemed reasonable to assume that when the ankle is sprained, rupture of nerve fibres leads to partial deafferentiation of the injured joint. ${ }^{19}$ However, the afferent fibres of most joint mechanoreceptors have a conduction velocity in a range of 3 to $70 \mathrm{~m} \mathrm{~s}^{-1}$, characteristic of groups II and III nerve fibre types. ${ }^{20}$ Receptors of group II and III units are also less sensitive to stretch than the muscle spindles and the Golgi tendon organs. ${ }^{21}$ It might therefore be assumed that the amount of muscle activity evoked by capsular and ligamentous proprioceptive inputs is relatively small and inefficient. ${ }^{22}$ Conversely, the conduction velocity of group I nerve fibres (the muscle spindle annulospiral endings and Golgi tendon organs) is $70-120 \mathrm{~m} \mathrm{~s}^{-1}$. Signals from group I receptors are important in minimising the consequences of small internal disturbances that occur during single leg standing. It should therefore be expected that postural instability would increase when inputs from these group I nerve fibre receptors are diminished. ${ }^{2}$

In conclusion, gymnasts' ability to control their stability on single leg standing over a chronically sprained ankle was found unaffected. This conclusion does not reject other theories of a partial proprioceptive deafferentiation of different receptors in the ankle joint which, however, might be replaced by afferent input from the calf muscles. ${ }^{18}$

This research was supported by the Fund for Promotion of Research at the Technion.

1 Han $\mathrm{KH}$, Muwanga CL. The incidence of recurrent soft tissue ankle injuries. Br f Clin Prac 1990;44:609-11.

2 Cass JR, Morrey BF. Ankle instability: current concepts, diagnosis, and treatment. Mayo Clin Proc 1984;59:165-70.

Smith RW, Reischl SF. Treatment of ankle spains in young athletes. Am ₹ Sports Med 1986;14:465-71.

4 Barrett DS, Cobb AG, Bentley G. Joint proprioception in normal, osteoarthritic and replaced knees. F Bone foint Surg Br 1991;73-B:53-6.

5 Newton RA. Joint receptor contributions to reflexive and kinesthetic responses. Phys Ther 1982;62:22-9.

6 Funder V, Jorgensen JP, Andersen A, Andersen SB, Lindholmer E, Niedermann B, Vuust $M$. Ruptures of the lateral ligaments of the ankle: clinical diagnosis. Acta Orthop Scand 1982;53:997-1000.

7 Rijke AM, Jones B, Vierhut PAM. Stress examination of traumatized lateral ligaments of the ankle. Clin Orthop Relat Res 1986;210:143-51.

8 Freeman M. Instability of the foot after injuries to the lateral ligament of the ankle. $\mathcal{F}$ Bone foint Surg $\mathrm{Br} 1965 ; 47-\mathrm{B}$ : 669-77.

9 Muwanga CL, Hellier M, Quinton DN, Sloan JP, Dove AF. Grade III injuries of the lateral ligaments of the ankle: the incidence and a simple stress test. Arch Emerg Med 1986;3: 247-51.

10 Freeman $M$, Dean $M$, Hanham I. The etiology and prevention of functional instability of the foot. $\mathcal{F}$ Bone foint Surg Br 1965;47-B:678-85.

11 Tropp H, Odenrick P, Gillquist J. Stabilometry recordings in functional and mechanical instability of the ankle joint. Int 7 Sports Med 1985;6:180-2.

12 Konradsen L, Raven JB. Ankle instability caused by prolonged peroneal reaction time. Acta Orthop Scand $1990 ; 61(5): 388-390$

13 Isakov E, Mizrahi J, Solzi P, Susak Z, Lotem M. Response of the peroneal muscles to sudden inversion of the ankle during standing. Int $\mathcal{F}$ Sport Biomech 1986;2:100-9.

14 Isakov E, Mizrahi J, Ring H, Susak Z, Hakim N. Standing sway and weight-bearing distribution in people with below-knee amputations. Arch Phys Med Rehabil 1992;73: 174-8.

15 Lentell GL, KatzmanLL, Walters MR. The relationship betwee muscle function and ankle stability. $\mathcal{F}$ Orthop Sport Phys Ther 1990;11:605-11.

16 Tropp $\mathrm{H}$. Pronator muscle weakness in functional instability of the ankle joint. Int $\mathcal{F}$ Sports Med 1986;7:291-4.

17 Brunt D, Andersen JC, Huntsman B, Reinhert LB, Thorell AC, Sterling JC. Postural responses to lateral perturbation in healthy subjects and ankle sprain patients. Med Sci in healthy subjects and ank
Sports $E x$ 1992;24:171-6.

18 Ekdahl C, Janlo GB, Andersson SI. Standing balance in healthy subjects. Scand f Rehab Med 1989;21:187-95.

19 Konradsen L, Raven JB, Sorensen AI. Proprioception at the ankle: the effect of anaesthetic blockade of the ligament receptors. F Bone foint Surg Br 1993;75-B:433-6.

20 Chusid JG. The spinal cord. In: Correlative neuroanatomy and functional neurology, 2nd ed. California: Lange Medica Publications, 1976:62-75.

21 Kniffki KD, Mense S, Schmidt RF. Muscle receptors with fine afferent fibers which may evoke circulatory reflexes. Circ Res 1981;48:25-31.

22 Evarts EV. Sherrington's concept of proprioception. Trends Neurol Sci 1981;2:44-6. 